\title{
Nutrition habits in 24-hour mountain bike racers
}

\author{
Daniela Chlíbková ${ }^{1 \dagger}$, Beat Knechtle $2,3,7^{*}$, Thomas Rosemann $^{2 \dagger}$, Ivana Tomášková ${ }^{4 \dagger}$, Vlastimil Chadim ${ }^{5 \dagger}$ \\ and Marcus Shortall ${ }^{6+}$
}

\begin{abstract}
We investigated seventy-four ultra-mountain bikers (MTBers) competing in the solo category in the first descriptive field study to detail nutrition habits and the most common food before during and after the 24 hour race using questionnaires. During the race, bananas (86.5\%), energy bars (50.0\%), apples (43.2\%) and cheese (43.2\%) were the most commonly consumed food, followed by bread (44.6\%), rice (33.8\%) and bananas (33.8\%) after the race. Average fluid intake was $0.5 \pm 0.2 \mathrm{l} / \mathrm{h}$. The main beverage was isotonic sports drink (82.4\%) during and pure water (66.2\%) after the race. The most preferred four supplements in the four weeks before, the day before, during and after the race were vitamin C (35.1\%), magnesium (44.6\%), magnesium (43.2\%) and branched-chain amino acids (24.3\%), respectively. Total frequency of food intake (30.6 \pm 10.5 times/24 hrs) was associated with fluid intake $(r=0.43, P=0.04)$ and both were highest at the beginning of the race and lower during the night hours and the last race segment in a subgroup of twenty-three ultra-MTBers. Supplement intake frequency (6.8 \pm 8.4 times/24 hrs) was highest during the night hours and lower at the beginning and end of the race. Elevated food and fluid intake among participants tracked across all race segments $(P<0.001)$. In conclusion, the nutrition strategy employed by ultra-MTBers was similar to those demonstrated in previous studies of ultra-cyclists with some exceptions among selected individuals.
\end{abstract}

Keywords: Race nutrition; 24-hour race; Ultra-cycling

\section{Background}

The continuous physical stress in ultra-endurance performances leads to an unavoidable energy deficit (Knechtle 2013). In most ultra-endurance exercises, energy intake is lower than energy expenditure (Bescós et al. 2012a, b; Bircher et al. 2006; Enqvist et al. 2010; Francescato et al. 2002; Linderman et al. 2004). Ultra-endurance sports such as 24-hour bike races require large caloric intakes in order to match the expenditures required to complete these prolonged events (Bescós et al. 2012a, b; Bircher et al. 2006; Francescato et al. 2002; Linderman et al. 2004; White et al. 1984). The type, timing and amount of foods ingested as well as the co-ingestion of ergogenic aids such as caffeine are important factors directly linked to sport performance in endurance events (Eberle 2007; Jeukendrup 2014; Laursen et al. 2001; Maughan et al. 2011; Ormsbee et al. 2014; Peters 2003). At present, comprehensive dietary macronutrient

\footnotetext{
* Correspondence: beat.knechtle@hispeed.ch

${ }^{\dagger}$ Equal contributors

${ }^{2}$ Institute of Primary Care, University of Zurich, Zurich, Switzerland

${ }^{3}$ Gesundheitszentrum St. Gallen, St. Gallen, Switzerland

Full list of author information is available at the end of the article
}

recommendations for ultra-endurance and adventure races are missing. As a result, race recommendations for carbohydrate (Burke 2002; Burke et al. 2011; Jeukendrup 2004, 2011, 2014; Lambert et al. 2003; Millard-Stafford et al. 2008; Peters 2003), protein (Ormsbee et al. 2014; Ranchordas 2012) and fat (Ormsbee et al. 2014; Lambert 2003) intake have been extrapolated from studies on traditional endurance events.

The nutritional strategy during a 24-hour race and a defined plan for feeding can be a key strategy to finish a race successfully (Burke 2002; Eberle 2007; Jeukendrup 2011). On the contrary, it is necessary to be flexible and open to changes if things are not working according to a defined plan. Many ultra-mountain bikers (ultra-MTBers) exhibit an over-reliance on sports supplements compared to traditional foodstuffs and/or they can expect to face extreme nutrient demands to achieve their fuel and fluid replacement goals. In events lasting $8-24$ hrs it is not necessary and practical to meet total energy expenditure (Burke 2002). The main goal is to match muscle fuel needs for carbohydrate (Burke 2002; Eberle 2007; Jeukendrup 2011; Stuempfle et al. 2011); however, there are many 
practical hurdles which must be overcome in order to achieve this goal (Burke 2002; Eberle 2007). In events such as triathlons or road cycling races it is easier to eat solid food while cycling. However, in a mountain bike race, the course is too technical to allow ample opportunity for feeding (Burke 2002). Competitors are more reliant on feed stations or food provided by their support crew or themselves at pit stops. Factors such as appetite suppression and gastrointestinal distress can also reduce the dietary intake during longer competition (Bescós et al. 2012a; Eberle 2007; Jeukendrup 2011, 2014).

Information about energy intake in ultra-endurance cyclists during a 24-hour race is scarce (Bescós et al. 2012a, b; Burke 2002; Knechtle et al. 2003; White et al. 1984). Most research conducted on nutrition during extreme endurance is based on case studies and studies involving a small number of individuals (Bescós et al. 2012a, b; Rehrer 2001; White et al. 1984). In relation to energy demands, several studies have assessed the nutritional requirements and behavior of cyclists during solo events (Francescato et al. 2002; Havemann et al. 2008; Knechtle et al. 2005; 2007; White et al. 1984). However, data on race diet in a solo category in a 24-hour mountain bike races are lacking. Therefore, the aim of this study was to investigate dietary, fluid and ergogenic aid intakes (1) one month prior, (2) one day prior, (3) during and (4) during the three hours following the race. We hypothesized that dietary intake and the use of supplements would not differ to current nutritional recommendations for long-distance events. Additionally, we evaluated the amount of fluid ingested, and the frequency of ingestion of solid food and supplements in four race segments during 24 hours. We predicted a lower fluid intake and a frequency of food intake during the night and a higher frequency of supplement intake in the last race segment before the end of the race.

\section{Methods}

Research within the project proceeded in an accordance with the law (No. 96/2001 Coll. M. S. on Human Rights and Biomedicine and Act No. 101/2000 Coll. Privacy) and the study was approved by the local institutional ethics committee (The Ethics Committee of the Faculty of Masaryk University, Brno, Czech Republic). UltraMTBers were notified approximately three months before the race of the study via an e-mail and were informed about the planned investigation with indication that participation is voluntary. Prior to the race, athletes were informed of the procedures and gave their informed written consent.

In the current study, we observed the nutritional habits of entrants in two 24-hour MTB races held in the Czech Republic in 2012. The first measurement was performed at the 'Czech Championship 24-hour MTB' in Jihlava (Race 1). The ultra-MTBers started on May 19th 2012 at 12:00 and finished on May 20th 2012 at 12:00. The course was primarily held on a $9.5 \mathrm{~km}$ single-track trail with an elevation of $220 \mathrm{~m}$. The second measurement was performed at the 24-hour race 'Bike Race Marathon MTB' in Liberec (Race 2) taking place from June 9th 2012 to June 10th 2012. The course was comprised of a $12.6 \mathrm{~km}$ loop with an elevation of $250 \mathrm{~m}$. Nearby temperature during both races ranged from $6^{\circ} \mathrm{C}$ to $23^{\circ} \mathrm{C}$. We acknowledge that the use of the temperatures from the nearest local weather stations underestimated the actual temperatures through some parts of the course. A single aid station was positioned at the start/finish area, where a variety of food and beverages such as hypotonic sports drinks, tea, soup, caffeinated drinks, water, fruit, vegetables, energy bars, bread, soup, sausages, cheese, bread, chocolate and biscuits were available. The ultra-MTBers could also use their own supplies during pre-determined breaks. In total, 77 racers consented to participate in the present study (i.e. 40 in Race 1 and 37 in Race 2).

Volunteers were asked to complete a training diary in the three-month period before the race. The training records consisted of sex, age, the number of years that they had actively participated in cycling, their best result (measured in $\mathrm{km}$ ) in a 24-hour race, the number of hours spent by training per week as well as the number of hours of cycling training per week. The MTBers also completed a questionnaire about their pre-race diet and the use of supplements before the race. Prior to the start of the race, a second questionnaire about race and post-race nutrition was administered to the participants by trained personnel. The MTBers and their support teams marked items and noted the amount of fluid and the frequency of food and supplements consumed at the aid station or provided by the support crew. For the purpose of recording, the race was broken into discrete segments (i.e. from start at $12.00 \mathrm{hrs}$ to $18.00 \mathrm{hrs}$, from $18.00 \mathrm{hrs}$ to $24.00 \mathrm{hrs}$, from $24.00 \mathrm{hrs}$ to $6.00 \mathrm{hrs}$ and from $6.00 \mathrm{hrs}$ to $12.00 \mathrm{hrs}$ the next day). The subjects were not told what food or fluid to consume during the race. During the event, the time and race speed taken by each athlete to complete each lap were recorded by a personal lap counter (Sportsoft timing spol. s.r.o. chip technology). After the race, the ultra-MTBers sent their completed questionnaires and reports by fax, postal letter or e-mail to the investigator. Participants were instructed to respond within ten days of the event.

\section{Statistical analyses}

The statistical software Statistica (StatSoft, Inc., Tulsa, OK USA) was used for the statistical analysis. Differences between pre-race variables (sex, age, years as an active biker, best result in a 24-hour race, average weekly 
training hours and average weekly cycle hours) in the racers from the two races in the subgroup $(n=23)$ were calculated using one-way ANOVA after fulfilling two basic presumptions - homogeneity of variance (Levene test) and the normality of distribution (Shapiro-Wilks test). The association between pre-race characteristics and the amount of fluid intake, the frequency of food or supplement intake was calculated using Spearmans rank correlation coefficient. Spearman correlation coefficient was used to reveal the measure of statistical dependence between race speed and fluid intake, between the frequency of food and/or supplements intake and the amount of fluid intake mutually, between total nutrition intake and intake in all race segments. Results in the text are presented as mean \pm SD. For all statistical tests, significance was set at a level of 0.05 .

\section{Results}

Seventy-four (96.1\%) of the 77 race participants successfully finished the race and returned their completed questionnaires. One MTBer was forced to retire due to an equipment failure, while two had medical complications. Fluid intake and the frequency of food and supplement intake were evaluated in a subgroup of 23 (13 males and 10 females) ultra-MTBers (30.1\%). Incomplete questionnaires were excluded from the final analysis. We found no significant differences in pre-race variables (i.e. sex, age, years as an active biker, best result in a 24-hour race, average weekly training hours and average weekly hours spent cycling per week) between the racers from two races in this subgroup $(P>0.05)$. Pre-race characteristics and an average race speed are presented in Table 1.

Pre-race dietary intake and supplementation $(n=74)$ In the four weeks preceding the race, only two ultraMTBers (2.7\%) followed no special pre-race diet (Table 2). Forty-seven racers $(63.5 \%)$ used a carbohydrate-rich diet and twenty-five athletes (34.8\%) accomplished a proteinand (or) fat-rich diet. Forty-five (60.8\%) racers consumed ergogenic supplements with a preference for branchedchain amino acids (BCAA) (21.6\%) and L-Carnitine (12.2\%). Fifty-one $(68.9 \%)$ racers ingested vitamins and favoured vitamin $\mathrm{C}(35.1 \%)$, multi-vitamin products
(18.9\%) and all kinds of vitamin B (14.9\%). Fifty (67.6\%) ultra-MTBers consumed minerals with a special preference for magnesium (32.4\%), multi-mineral products (13.5\%) and potassium (10.8\%) (Table 3$)$.

The day before the race, all ultra-MTBers followed a planned dietary routine, sixty-two (83.8\%) racers accomplished a carbohydrate-rich and twelve racers (16.2\%) a protein-rich diet. Twenty-five (33.8\%) ultra-MTBers consumed ergogenic supplements with a preference for BCAA (17.6\%) and L-Carnitine (10.8\%). Thirty-nine (52.7\%) racers ingested vitamins and favoured vitamin $\mathrm{C}(31.1 \%)$, multi-vitamin products (12.2\%) and B vitamins (12.2\%). Forty-nine (66.2\%) ultra-MTBers consumed minerals with a special preference for magnesium (44.6\%) and multimineral products $(12.2 \%)$ (Table 3$)$.

\section{In-race dietary intake and supplementation $(n=74)$}

A variety of fifty-six different solid foods and sixteen beverages were consumed during the race (Tables 4 and 5). The ultra-MTBers have achieved an average fluid intake $0.5 \pm 0.2 \mathrm{l} / \mathrm{h}(12.0 \pm 5.0 \mathrm{l} / 24 \mathrm{hrs})$. The main beverage during the race was isotonic sports drink (82.4\%), followed by pure water (71.6\%), Coca Cola $(54.1 \%)$, and tea (51.4\%) (Table 5). The highest intake was $22.0 \mathrm{l} / 24 \mathrm{hrs}$ and the lowest intake was 5.1 l/24 hrs (Figure 1 ). The highest intake we recorded during the first segment of the race [S1 (i.e. $12.00-18.00 \mathrm{hrs}$ ) $3.5 \pm 1.5 \mathrm{l}$, respectively $0.6 \pm$ $0.2 \mathrm{l} / \mathrm{h}$ ] with an average fluid consumption decreasing during each subsequent segment [S2 (i.e. 18.00 - $24.00 \mathrm{hrs}$ ) $3.1 \pm 1.4 \mathrm{l}$ equal to $0.5 \pm 0.3 \mathrm{l} / \mathrm{h}, \mathrm{S} 3$ (i.e. $24.00-6.00 \mathrm{hrs}$ ) $2.7 \pm 1.3 \mathrm{l}$, respectively, $0.5 \pm 0.3 \mathrm{l} / \mathrm{h}, \mathrm{S} 4$ (i.e. 6.00 $12.00 \mathrm{hrs}) 2.6 \pm 1.2 \mathrm{l}$ equal to $0.4 \pm 0.2 \mathrm{l} / \mathrm{h}$. Racers were assigned to the three groups based on their race speed (group 1A: MTBers with a race speed of $12.8-14.8 \mathrm{~km} / \mathrm{h}$, group 1B: 14.9 - $16.9 \mathrm{~km} / \mathrm{h}$ and group 1C: $17.0-19.0 \mathrm{~km} / \mathrm{h}$ ) (Figure 2). Average fluid intake in group $1 \mathrm{~A}$ was $0.6 \pm 0.1 \mathrm{l} / \mathrm{h}$, in group $1 \mathrm{~B} 0.4 \pm 0.1 \mathrm{l} / \mathrm{h}$ and in group $1 \mathrm{C} 0.6 \pm 0.1 \mathrm{l} / \mathrm{h}$. We found no association between race speed and the amount of fluid intake in the present 24-hour ultraMTBers $(P>0.05)$ (Figure 2).

Ultra-MTBers ate with an average frequency $30.6 \pm 10.5$ times/24 hrs, equally to $1.3 \pm 0.4$ times/h. Bananas $(86.5 \%)$ were preferred before energy bars (50.0\%), apples (43.2\%),

Table 1 The pre-race and race characteristics of the soubgroup $(n=23)$

\begin{tabular}{lcccc}
\hline Parameter & Unit & All ultra-MTBers $(\boldsymbol{n}=\mathbf{2 3})$ & Male ultra-MTBers $(\boldsymbol{n}=\mathbf{1 3})$ & Female ultra-MTBers $(\boldsymbol{n}=\mathbf{1 0})$ \\
\hline Age & yrs & $36.3 \pm 6.6$ & $35.4 \pm 6.5$ & $37.3 \pm 6.6$ \\
Years as an active biker & $\mathrm{yrs}$ & $7.6 \pm 2.9$ & $8.0 \pm 3.0$ & $7.2 \pm 2.7$ \\
Personal best average km in a 24-hour race & $\mathrm{km}$ & $280.8 \pm 87.2$ & $313.6 \pm 82.9$ & $238.1 \pm 73.1$ \\
Training hours weekly & $\mathrm{h}$ & $11.3 \pm 3.6$ & $10.6 \pm 4.3$ & $12.3 \pm 2.2$ \\
Cycling hours weekly & $\mathrm{h}$ & $10.0 \pm 3.2$ & $9.7 \pm 3.8$ & $10.5 \pm 2.0$ \\
Race speed & $\mathrm{km}$ & $15.8 \pm 1.7$ & $16.5 \pm 1.7$ & $15.3 \pm 1.3$ \\
\hline
\end{tabular}

Note: data are presented as mean \pm standard deviation. 
Table 2 Diet in four weeks and the day before the start of race $(n=74)$

\begin{tabular}{lcccc}
\hline Kind of diet & $\begin{array}{c}\text { Four weeks } \\
\text { before } \\
\text { the race }\end{array}$ & Percentage (\%) & $\begin{array}{c}\text { The day } \\
\text { before } \\
\text { the race }\end{array}$ & Percentage (\%) \\
\hline $\begin{array}{l}\text { Carbohydrate- } \\
\text { rich diet }\end{array}$ & 47 & 63.5 & 62 & 83.8 \\
$\begin{array}{l}\text { Protein-and (or) } \\
\text { fat-rich diet }\end{array}$ & 25 & 33.8 & 12 & 16.2 \\
$\begin{array}{l}\text { No special diet } \\
\text { nof }\end{array}$ & 2 & 2.7 & 0 & 0 \\
\hline
\end{tabular}

cheese (43.2\%), biscuits (40.5\%) and bread (37.8\%) (Table 4). The racer with the highest frequency ate 48 times and the racer with the lowest frequency ate 16 times in the 24 hours (Figure 1). Racers ate the most $(9.3 \pm 4.9$ times) during the first race segment (i.e. 12.00 - $18.00 \mathrm{hrs)}$, equal to $1.6 \pm 0.8$ times/h; with a decrease $(6.9 \pm 3.8$ times $)$ during the second segment (i.e. 18.00 - $24.00 \mathrm{hrs}$ ), $1.1 \pm$ 0.6 times/h, respectively. A moderate increase appeared during the third segment (i.e. 24.00 - $6.00 \mathrm{hrs)} 7.0 \pm 3.5$ times, equal to $1.2 \pm 0.6$ times/h and during the last fourth segment (i.e. $6.00-12.00 \mathrm{hrs)} 7.3 \pm 3.6$ times, equal to $1.2 \pm 0.6$ times/h, respectively.

BCAA were the preferred ergogenic supplement during the race for twenty-two athletes (29.7\%), and L-Carnitine for fifteen racers (20.3\%). Thirty-two (43.2\%) racers

Table 3 Supplementation before, during and after the race $(n=74)$

\begin{tabular}{|c|c|c|c|c|c|c|c|c|}
\hline $\begin{array}{l}\text { Kind of } \\
\text { supplementation }\end{array}$ & $\begin{array}{c}\text { Four weeks } \\
\text { before the race }\end{array}$ & $\begin{array}{c}\text { Percentage } \\
\text { (\%) }\end{array}$ & $\begin{array}{c}\text { The day } \\
\text { before the race }\end{array}$ & $\begin{array}{c}\text { Percentage } \\
(\%)\end{array}$ & $\begin{array}{l}\text { During the } \\
\text { race }\end{array}$ & $\begin{array}{c}\text { Percentage } \\
\text { (\%) }\end{array}$ & $\begin{array}{l}\text { After the } \\
\text { race }\end{array}$ & $\begin{array}{c}\text { Percentage } \\
\text { (\%) }\end{array}$ \\
\hline \multicolumn{9}{|l|}{$\begin{array}{l}\text { Ergogenic } \\
\text { supplements }\end{array}$} \\
\hline Concentrate of $\mathrm{AA}$ & 5 & 6.8 & 3 & 4.1 & 5 & 6.8 & 5 & 6.8 \\
\hline BCAA & 16 & 21.6 & 13 & 17.6 & 22 & 29.7 & 18 & 24.3 \\
\hline Coenzym Q10 & 5 & 6.8 & 4 & 5.4 & 1 & 1.4 & 2 & 2.7 \\
\hline L-Carnitine & 9 & 12.2 & 8 & 10.8 & 15 & 20.3 & 4 & 5.4 \\
\hline Glutamine & 4 & 5.4 & 2 & 2.7 & 2 & 2.7 & 1 & 1.4 \\
\hline Concentrate of EFA & 5 & 6.8 & 2 & 2.7 & 2 & 2.7 & 1 & 1.4 \\
\hline Caffeine tablets & 0 & 0 & 0 & 0 & 2 & 2.7 & 0 & 0 \\
\hline Lecithin & 0 & 0 & 0 & 0 & 1 & 1.4 & 0 & 0 \\
\hline Glucose & 0 & 0 & 0 & 0 & 3 & 4.1 & 0 & 0 \\
\hline Creatine & 4 & 5.4 & 3 & 4.1 & 5 & 6.8 & 1 & 1.4 \\
\hline No intake of ES & 29 & 39.2 & 49 & 66.2 & 18 & 24.3 & 46 & 62.2 \\
\hline \multicolumn{9}{|l|}{ Vitamins } \\
\hline Multi-vitamin & 14 & 18.9 & 9 & 12.2 & 8 & 10.8 & 8 & 10.8 \\
\hline Vitamin C & 26 & 35.1 & 23 & 31.1 & 9 & 12.2 & 13 & 17.6 \\
\hline Vitamin B (complex) & 11 & 14.9 & 9 & 12.2 & 5 & 6.8 & 9 & 12.2 \\
\hline Vitamin E & 3 & 4.1 & 3 & 4.1 & 2 & 2.7 & 4 & 5.4 \\
\hline Folic acid & 2 & 2.7 & 2 & 2.7 & 2 & 2.7 & 2 & 2.7 \\
\hline No intake of vitamins & 23 & 31.1 & 35 & 47.3 & 57 & 77.0 & 51 & 68.9 \\
\hline \multicolumn{9}{|l|}{ Minerals } \\
\hline Multi-mineral & 10 & 13.5 & 7 & 9.5 & 8 & 10.8 & 3 & 4.1 \\
\hline Magnesium & 24 & 32.4 & 33 & 44.6 & 32 & 43.2 & 14 & 18.9 \\
\hline Calcium & 7 & 9.5 & 3 & 4.1 & 5 & 6.8 & 4 & 5.4 \\
\hline Iron & 5 & 6.8 & 1 & 1.4 & 0 & 0 & 1 & 1.4 \\
\hline Zinc & 5 & 6.8 & 4 & 5 & 3 & 4.1 & 1 & 1.4 \\
\hline Salt tablets & 0 & 0 & 0 & 0 & 6 & 8.1 & 1 & 1.4 \\
\hline Potassium & 8 & 10.8 & 3 & 4.1 & 4 & 5.4 & 3 & 4.1 \\
\hline No intake of minerals & 24 & 32.4 & 25 & 33.8 & 18 & 24.3 & 60 & 81.1 \\
\hline
\end{tabular}

Note: concentrate of AA - concentrate of amino acid, BCAA - branched-chain amino acid, concentrate of EFA - concentrate of Essentials fatty acid, no intake of ES - no intake of ergogenic supplements. 
Table 4 Food intake during and after the race $(n=74)$

\begin{tabular}{lcccc}
\hline Kind of food & $\begin{array}{c}\text { During } \\
\text { the race }\end{array}$ & $\begin{array}{c}\text { Percentage } \\
(\%)\end{array}$ & $\begin{array}{c}\text { After the } \\
\text { race }\end{array}$ & $\begin{array}{c}\text { Percentage } \\
(\%)\end{array}$ \\
\hline
\end{tabular}

Fruits and nuts

Bananas

Apples

Oranges

Raisins

Grapes

Peaches

Blueberries

Apricots

Peanuts

Pistachio nuts

Sunflower seeds

Pears

Dried fruits

Figs

Strawberries

Pineapple

Cherries

Walnuts

Melon

Kiwi

Fruit purée

Grapefruit

Meat and fish

Chicken

Fish

Beef

Pork

Sausages

White pudding

Vegetables and

salads

Potatoes

Beans

Carrots

Corn

Cucumbers

Tomatoes

Olives

Soy

Vegetables sushi

Zucchini

Oyster mushroom

Pickles

86.5
43.2
24.3
20.3
10.8
27

Table 4 Food intake during and after the race $(n=74)$ (Continued)

\begin{tabular}{|c|c|c|c|c|}
\hline \multicolumn{5}{|l|}{$\begin{array}{l}\text { Carbohydrate-rich } \\
\text { food }\end{array}$} \\
\hline Bread & 28 & 37.8 & 33 & 44.6 \\
\hline Noodles & 19 & 25.7 & 23 & 31.1 \\
\hline Rice & 21 & 28.4 & 25 & 33.8 \\
\hline Carbohydrate gel & 10 & 13.5 & 0 & 0 \\
\hline Cake & 2 & 2.7 & 2 & 2.7 \\
\hline Biscuits & 30 & 40.5 & 11 & 14.9 \\
\hline Pizza & 0 & 0 & 1 & 1.4 \\
\hline Chips & 2 & 2.7 & 6 & 8.1 \\
\hline Cornflakes & 1 & 1.4 & 1 & 1.4 \\
\hline Porridge & 7 & 9.5 & 5 & 6.8 \\
\hline Energy bars & 37 & 50.0 & 10 & 13.5 \\
\hline Muesli bars & 10 & 13.5 & 0 & 0 \\
\hline Soy bars & 2 & 2.7 & 0 & 0 \\
\hline Pancakes & 2 & 2.7 & 0 & 0 \\
\hline Honey & 2 & 2.7 & 0 & 0 \\
\hline Treacle & 1 & 1.4 & 0 & 0 \\
\hline \multicolumn{5}{|l|}{ Dairy produce } \\
\hline Cheese & 32 & 43.2 & 18 & 24.3 \\
\hline Eggs & 1 & 1.4 & 5 & 6.8 \\
\hline Yoghurt & 0 & 0 & 8 & 10.8 \\
\hline Ice team & 0 & 0 & 5 & 6.8 \\
\hline Chocolate & 19 & 25.7 & 10 & 13.5 \\
\hline Cottage cheese & 1 & 1.4 & 0 & 0 \\
\hline Pudding & 1 & 1.4 & 0 & 0 \\
\hline Sour team & 0 & 0 & 1 & 1.4 \\
\hline
\end{tabular}

consumed magnesium, nine (12.2\%) consumed vitamin C, five (6.8\%) B vitamins, and eight (10.8\%) multi-mineral products (Table 3 ). The ultra-MTBers supplemented $6.8 \pm 8.4$ times $/ 24$ hrs (Figure 1 ). The highest achieved frequency by one racer was $33 \pm 10.2$ times $/ 24$ hrs, the lowest intake of supplements was zero times/24 hrs. Supplements were consumed $1.4 \pm 2.2$ times during the first segment (i.e. 12.00 - $18.00 \mathrm{hrs}$ ), $2.0 \pm 2.3$ times during the second segment (i.e. $18.00-24.00 \mathrm{hrs}$ ), $1.9 \pm 2.6$ times during the third segment (i.e. $24.00-6.00 \mathrm{hrs}$ ) and $1.7 \pm 2.2$ times during the final race segment (i.e. 6.00 $12.00 \mathrm{hrs})$.

Post-race dietary intake and supplementation $(n=74)$ Forty-three different solid foods and eighteen different beverages were consumed after the race (Tables 4 and 5). The preferred food was bread (44.6\%), followed by rice (33.8\%), bananas (33.8\%), chicken (33.8\%), noodles (31.1\%), 
Table 5 Beverages during and after the race $(n=74)$

\begin{tabular}{lcccc}
\hline Beverage & $\begin{array}{c}\text { During the } \\
\text { race }\end{array}$ & $\begin{array}{c}\text { Percentage } \\
\text { (\%) }\end{array}$ & $\begin{array}{c}\text { After the } \\
\text { race }\end{array}$ & $\begin{array}{c}\text { Percentage } \\
\text { (\%) }\end{array}$ \\
\hline Water & 53 & 71.6 & 49 & 66.2 \\
Isotonic sports & 61 & 82.4 & 20 & 27.0 \\
drink & & 23.0 & 13 & 17.6 \\
Coffee & 17 & 6.8 & 3 & 4.1 \\
Apple juice & 5 & 54.1 & 27 & 36.5 \\
Coca Cola & 40 & 13.5 & 29 & 39.2 \\
Beer & 10 & 51.4 & 19 & 25.7 \\
Tea & 38 & 45.9 & 22 & 29.7 \\
Soup & 34 & 1.4 & 4 & 5.4 \\
Orange juice & 1 & 2.7 & 5 & 6.8 \\
Lemonade & 2 & 1.4 & 2 & 2.7 \\
Ice tea & 1 & 1.4 & 4 & 5.4 \\
Milk & 1 & 1.4 & 2 & 2.7 \\
Chocolate milk & 1 & 1.4 & 2 & 2.7 \\
Cocktail & 1 & 5.4 & 4 & 5.4 \\
Fruit juice & 4 & 0 & 1 & 1.4 \\
(other) & & 27.0 & 4 & 5.4 \\
Tomato juice & 0 & 0 & 5 & 6.8 \\
Red Bull & 20 & & & \\
Regener drink & 0 & & & \\
\hline
\end{tabular}

cheese $(24.3 \%)$, pork $(18.9 \%)$, and potatoes $(17.6 \%)$. The main beverage was pure water $(66.2 \%)$, followed by beer (39.2\%), Coca Cola (36.5\%) and soup (29.7\%). BCAA were the most preferred ergogenic supplement (24.3\%); vitamin C (17.6\%) and magnesium (18.9\%) were preferably consumed as vitamins and minerals after the race (Table 3).

The total amount of fluid ingested was related to fluid intake in each race segment (S1: $r=0.88, P<0.001$; S2: $r=0.91, P<0.001 ; \mathrm{S} 3: r=0.92, P<0.001 ; \mathrm{S} 4: r=0.76$, $P<0.001$ ). Also the total supplement frequency (S1: $r=0.91$, $P<0.001$; S2: $r=0.84, P<0.001$; S3: $r=0.93, P<0.001$; S4: $r=0.89, P<0.001)$ and the food intake frequency (S1: $r=0.61, P<0.001 ; \mathrm{S} 2: r=0.79, P<0.001 ; \mathrm{S} 3: r=0.74$, $P<0.001$; S4: $r=0.49, P<0.001)$ associated with frequencies in all race segments. Fluid intake was related to the frequency of food intake $(\mathrm{r}=0.48, P<0.05)$, but not related to the frequency of supplement intake $(P>0.05)$. Food intake did not associate with the frequency of supplement intake $(P>0.05)$. We found no significant differences between pre-race variables (sex, age, years as an active biker, best result in a 24-hour race, average weekly training hours and average weekly cycle hours) and the amount of fluid intake, the frequency of food intake, or the frequency of supplement intake $(P>0.05)$. The highest correlation appeared between the amount of fluid intake and the participants best result in a 24-hour race $(r=0.24)$, however, the correlation was not significant $(P>0.05)$.
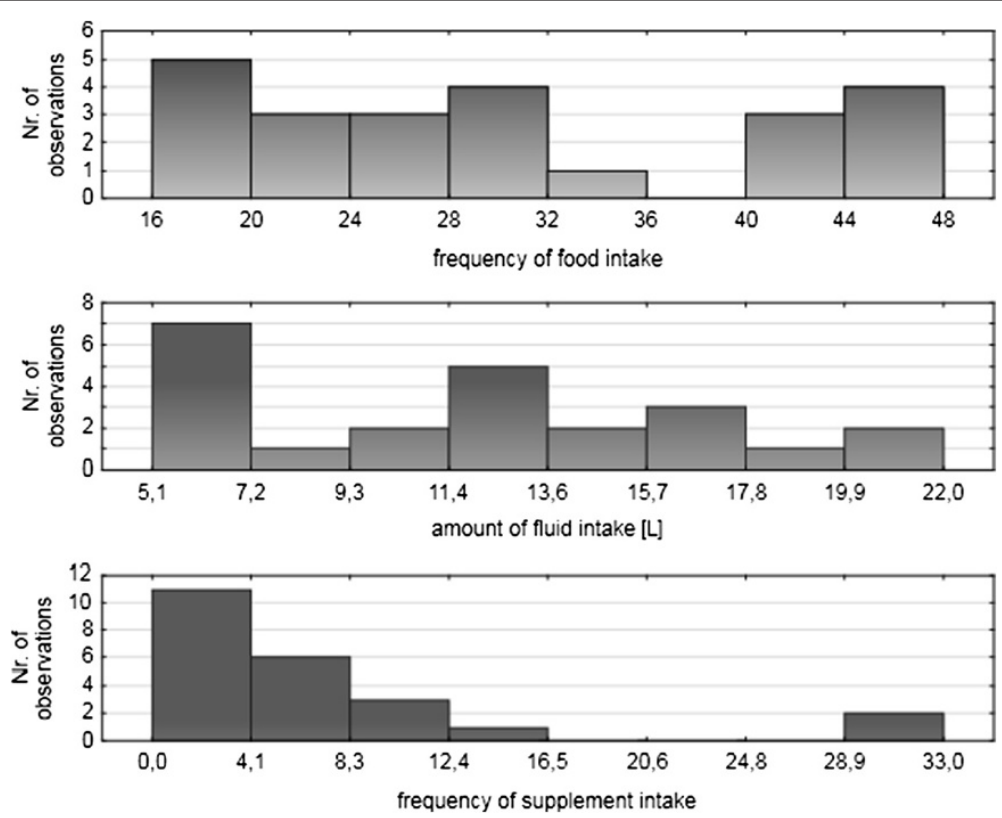

Figure 1 The frequency of food intake during 24-hour mountain bike race. The amount of fluid intake during 24-hour mountain bike race. The frequency of supplement intake during 24-hour mountain bike race. Note: number of testing subjects $(n=23)$. 


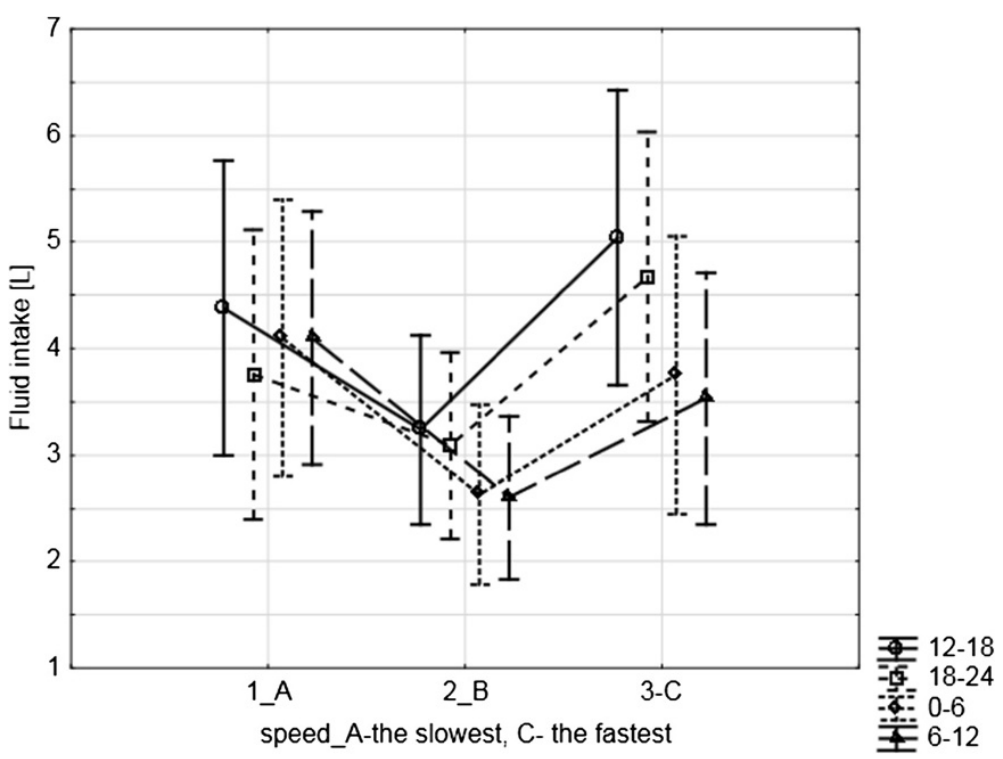

Figure 2 Fluid intake and race speed. Note: group 1A - MTBers with race speed $12.80-14.80 \mathrm{~km} / \mathrm{h}$, group 1 B - MTBers with race speed $14.90-16.90 \mathrm{~km} / \mathrm{h}$ and group $1 \mathrm{C}$ - MTBers with race speed $17.00-19.00 \mathrm{~km} / \mathrm{h}$. Race segments: 1. $12.00-18.00 \mathrm{hrs}, 2.18 .00-24.00 \mathrm{hrs}$, 3. $24.00-6.00$ hours, $4.6 .00-12.00$ hrs. Note: number of testing subjects $(n=23)$.

\section{Discussion}

Pre-race dietary intake did not differ from current nutritional recommendations for long-distance events. However, not all present ultra-MTBers followed a strict carbohydrate-rich diet before the race. Nevertheless, most of the athletes in the current study tried to achieve high concentrations in muscle glycogen prior to the start of the race by the traditional supercompensation protocol, while, a portion of the participants followed protein- and fat-rich diets. Having elevated muscle glycogen stores at the start may be beneficial for endurance performance, although this does not necessarily have to be achieved by the traditional supercompensation diet (Jeukendrup 2011). Well-trained endurance athletes can achieve a glycogen supercompensation without the need for the depletion phase prior to loading (Burke et al. 2011; Kreider 1991) and a higher carbohydrate intake might not always result in a better performance (Jeukendrup 2011). In a study by Knechtle et al. (2007), fifty percent of the successful finishers did not follow a carbohydrate-rich diet prior to competing in the, Race across America (RAAM).

No consistent performance benefit has been shown following a high-fat diet prior to an endurance performance (Ormsbee et al. 2014; Peters 2003). Interventions involving increased fat availability have failed to demonstrate ergogenic benefits in performance despite reduced carbohydrate utilization during exercise, increased free fatty acid levels in the blood and an increased lipid metabolism during exercise (Hargreaves et al. 2004; Lambert et al. 1994; Phinney et al. 1983). Dietary fat intake over a 24-hour period increased muscle triglyceride stores, but reduced cycling time-trial performance in seven endurance-trained men completing a 120-min cycling bout, compared with a high carbohydrate diet (Starling et al. 1997). The effects on subsequent exercise performance are equivocal (Ormsbee et al. 2014) and may show benefits for moderate-intensity in an ultra-distance setting, which favors fat as the primary fuel source (Hawley et al. 1995). Carbohydrate and protein ingestion prior to exercise could potentially enhance exercise performance in humans by augmenting and/or sparing glycogen stores (Rowlands and Hopkins 2002). Conversely, despite substantial effects on plasma hormone concentrations and fuel utilization, high-fat, high-protein and high-carbohydrate pre-exercise meals have no clear effect on cycling performance (Rowland et al. 2002). Nevertheless, current evidence continues to support mandatory high carbohydrate intakes before an event to maximize muscle and liver glycogen stores (Peters 2003). Carbohydrate intakes prior to endurance exercise have generally been shown to enhance performance, despite increasing insulin levels and reducing fat oxidation (Ormsbee et al. 2014).

Fluid and food intake was similar to common nutrition habits of ultra-cyclists with some exceptions in the current study. Dietary intake was highest at the beginning of the race and lower during night hours, in the middle of the race (the second and the third race segments) and at the end of the race (the last race segment). In the ultra-MTBers with the highest dietary intake, this characteristic tracked across all four segments. Average fluid intake was in an accordance with the International Marathon medical Directors Association (Hew-Butler 
et al. 2006). The present ultra-MTBers preferred carbohydrate calories originating from fruits (e.g. bananas, apples, oranges and raisins), from energy beverages (e.g. isotonic sports drinks and Coca Cola), from energy bars, carbohydrate gels, or from other carbohydrate-rich foods such as biscuits and bread. The most frequently consumed food in the present ultra-MTBers were bananas, which was in an agreement with Knechtle et al. study about the ultra-cycling race, RAAM' (Knechtle et al. 2007). Cheese, a food that contains more dietary fat than carbohydrate, was the third most ingested food during the present 24-hour race.

After the race, the most consumed food was bread, followed by chicken, with apples being the third preferred food in the present study. Carbohydrates are considered to be the most important fuel during ultra-endurance exercise and it is essential to ingest carbohydrate (Applegate 1991; Burke et al. 2001; Cermak and van Loon 2013; Jeukendrup 2010, 2011, 2014; Kreider 1991; Pfeiffer et al. 2012; Stellingwerff and Cox 2014). Nevertheless, cheese was also mentioned as a favorite food during the race and chicken was preferred even before bread in contrast to the present study in the ultra-endurance road cyclists competing in the, RAAM' (Knechtle et al. 2007). The energy demands of a 24-hour cycling event under laboratory conditions (White et al. 1984) represented near maximal levels of sustainable ergogenic effort by a cyclist in a case study. Most of the carbohydrate intake during an Ironman triathlon occurred during the cycling leg, where intake was almost three times as high as during the running leg (Kimber et al. 2002). Pfeiffer et al. (2012) demonstrated that higher carbohydrate intakes occur in cycling and triathlon events compared marathons and that better performance in Ironman races correlated with greater carbohydrate intake. With carbohydrate feeding during cycling, it has repeatedly been shown that muscle glycogen breakdown is unaffected (Jeukendrup 2011). Performance nutrition is based on evidence that increased carbohydrate availability enhances endurance and performance (Burke et al. 2001; Cermak and van Loon 2013; Jeukendrup 2014; Pfeiffer et al. 2012). The availability of carbohydrate as a substrate for muscle and the central nervous system is an important factor in endurance performances enduring for longer than 90 min (Burke et al. 2001). On the contrary, the role of protein metabolism during ultra-endurance race is not clear (Kreider 1991; Rowland et al. 2002). Endurance exercise results in the oxidation of several amino acids; however, the total amount of amino acid oxidation during endurance performance amounts to only 1-6\% of the total energy cost of exercise (Tarnopolski 2004). A higher protein consumption can be associated with a reduction of food intake and an increase of the risk of gastrointestinal disturbances (Jeukendrup 2011, 2014; Pfeiffer et al. 2012). In the study of Saunders et al. (2004), a carbohydrate beverage with additional protein calories produced significant improvements in fifteen male cyclists riding on a cycle ergometer in time to fatigue and reductions in muscle damage. However, it was not clear whether these effects were the result of the higher total calorie content of the beverage or due to specific protein-mediated mechanisms. Some, but not all (Breen et al. 2010), studies investigating the effects of post-exercise carbohydrate-protein intake on exercise performance have also noted an enhanced performance (Berardi et al. 2008), possibly as a result of an increased glycogen resynthesis (Berardi et al. 2006). Most of the present ultra-MTBers commercial carbohydrate drinks like the cyclists in the, RAAM' (Clark et al. 1992), but not in study by Knechtle et al. (2007), where they preferred pure water. The main beverage differed during the race, the ultra-MTBers preferred isotonic sports drinks, and after the race, the athletes preferred pure water. In a study perfomed by Clark et al. (1992), the road cyclists also preferred concentrates of carbohydrates such as carbohydrate drinks and sports bars during the race. The use of multiple transportable carbohydrates is beneficial in prolonged exercise, nevertheless with individual exceptions (Stellingwerff and Cox 2014).

Our hypothesis that fluid intake would be lower during the night and the last segment of the race was confirmed. On the contrary, we did not found a relationship between fluid intake and race speed in present ultraMTBers. The frequency of solid food was similarly highest during the first segment of the race. The lowest frequency of fluid intake was at the end of the race, food intake frequency was lowest during the second segment of the race; however, with only moderate differences in the last two segments. The decrease in food and fluid intake in some race segments in this study may have been attributable to fatigue, or gastrointestinal distress (Burke et al. 2011). This theory can be supported by the positive association between the fluid amount and the frequency of food intake. These results suggest that ultra-MTBers who had no problems ingesting food were also more likely to drink more and conversely, those with nausea, problems with food intake, lack of support team were more likely to have not eaten and drank less. Notwithstanding, racers adhered to their diet plan during all race segments, with decreasing amounts of food and drink being ingested during night hours and towards the end of the race. Other reasons for decreased dietary intake could also have been lack of familiarity with feeding at night and also altered biological rhythms associated with being awake for more than twenty four hours. Supplement intake was highest in the second and the third race segments, during the night hours, in the middle of the race, which was the inverse of food and fluid intake. We hypothesized the possibility of the replacement of solid 
food and fluid by ergogenic supplements in the present ultra-MTBers. However, we found no significant associations between the supplement frequency and the amount of fluid intake or the frequency of food intake. We presumed that the higher frequency of supplement intake in the last race segment before the end of the race could be attributed to possible nausea and problems associated with eating at that time. The hypothesis was not confirmed, all intakes (fluid, food and supplement) were lower during the last race segment and racers were presumably too tired to keep any dietary guidelines. We observed large differences in the amount of fluid ingested as well as food and supplement frequency among the individual ultraMTBers. For example, food frequency was three times higher and supplement intake was even thirty-three times higher in some cases than in other ones during the race. On the contrary, an interesting finding was the fact that thirty-nine percent of the racers in the four weeks before the race, sixty-six percent the day before, twenty-four percent during and sixty-two percent after the race did not use any ergogenic supplements. Finally, some competitors entered the race without a prepared fuelling protocol and according to Burke (2002) next reason could be various race nutrition plan or MTBers without any diet plan. Athletes who are racing to win have different nutrition strategies compared to those who are competing to finish the race (Burke 2002).

The most preferred supplements prior, during and after the 24-hour MTB race were vitamin $C$, magnesium and branched-chain amino acids. Intake of supplements such as vitamin $\mathrm{C}$, multi-vitamin, vitamin $\mathrm{B}$ (complex) and minerals (e.g. magnesium and multi-mineral products) is widespread in athletes (Frohnauer et al. 2008; Singh et al. 1993). In a study investigating Triple Iron ultra-triathletes, there was no association between race performance and vitamin and mineral ingestion in the four weeks leading up to the race. In studies by Frohnauer et al. (2008) and Knechtle et al. (2008), ultra-runners with a regular intake of vitamin and mineral supplements in the four weeks before the race finished the competition no faster than athletes without intake of vitamins and minerals. Moreover, in recent studies vitamin $\mathrm{C}$ supplementation decreased training efficiency in runners because it prevented some cellular adaptations to exercise (Gomez-Cabrera et al. 2008). The results of a study in endurance runners by Paulsen et al. (2014) showed that vitamin C and E supplements blunted elevations in mitochondrial protein content, which is needed for improving muscular endurance. Furthermore, $\mathrm{VO}_{2} \max$ and running performance were not detectably affected by the supplementation, and the authors pointed out that high dosages of vitamin $C$ should be used with caution. The preferred supplement the day before and during the race in the present study was magnesium. Magnesium supplementation, in general, does not enhance sport performance in well-nourished athletes (Nielsen and Lukaski 2006; Williams 2005), however, marginal magnesium deficiency has been shown to impair performance and amplify the negative consequences of strenuous exercise. The most consumed supplement among present ultra-MTBers after the race were branchedchain amino acids. No studies have attempted to identify levels of BCAA intake that might produce adverse effects on the brain (Fernstrom 2005). Several studies reported a reduced increase in myocellular enzymes, plasma creatine kinase (Saunders et al. 2007) and myoglobin (Valentine et al. 2008) in cyclists after the combined ingestion of carbohydrates and protein. The present ultra-MTBers probably chose to ingest BCAA to enhance their performance (Matsumoto et al. 2009; Tarnopolsky 2004), to protect their muscle mass (Shimomura et al. 2004) and to promote synthesis of muscle protein (Shimomura et al. 2004). During renal failure, an oral supplementation of BCAA can improve appetite and nutritional status (Cano et al. 2006). Pre-race BCAA supplementation may increase subsequent competitive performance when ingested for a longer period of time high-intensity resistance training (overreaching) (Sharp et al. 2010). In contrast, Knechtle et al. (2012) concluded that BCAA-supplementation before and during a $100-\mathrm{km}$ ultra-marathon had no effect on performance, skeletal muscle damage or renal function. Although BCAA also have biochemical and functional effects on the brain, few attempts have been made to characterize time-course or dose-response relations for such effects (Fernstrom 2005).

The third most drank beverage during and after the race in the present study was Coca Cola. Little is known about the intake of ergogenic supplements and their effect on ultra-endurance performance (Singh et al. 1993). In addition, only a few supplements have been evidenced to enhance performance in the absence of harm (Maughan et al. 2011). The ergogenic potential of caffeine on endurance performance lasting approximately one hour has been well documented (Burke 2008, Conway et al. 2003, Cox et al. 2002, Desbrow et al. 2012, Ganio et al. 2009, Goldstein et al. 2010; Meeusen et al. 2013; Pink et al. 2010) and recent research on the effects of pre-exercise caffeine consumption generally supported it as an ergogenic aid (Ormsbee et al. 2014). Caffeine does not improve maximal oxygen capacity directly, but could permit the athlete to train longer in activities lasting longer than two hours. Caffeine intake during sport events has become popular and practical, athletes use ordinarily specialized foods like sports drinks and gels with caffeine and carbohydrates and replacing sports drink with Coca-Cola ${ }^{\circ}$ during the latter stages of exercise is equally effective in enhancing endurance performance (Cox et al. 2002). The performance-enhancing effects of caffeine reside in the brain, although more research is necessary to reveal the 
exact mechanisms through which the central nervous system effect is established (Meeusen et al. 2013). An intake of caffeine before and also during exercise improves cycling performance (Conway et al. 2003; Cox et al. 2002; Desbrow et al. 2012). The competitive inhibition of adenosine receptors and subsequent central nervous system stimulation may explain the reported suppressed feelings of discomfort and pain experienced (Astorino et al. 2012) and the attenuated ratings of perceived exertion (RPE) (Stadheim et al. 2013) during exercise with pre-exercise caffeine consumption. Caffeine in the form of sugar sweetened carbonated beverages (Coca Cola ${ }^{\circ}$, Red Bull) and carbohydrate bars and gels was therefore a commonly used ergogenic supplement during and after the race in the current study.

\section{Limitations}

The limitation of the study is the use of the self-perception of the athletes to define the type of diet followed in the 3 month period prior to the race.

\section{Conclusions}

The dietary intake and use of supplements seen in the present study were similar to common recommendations for ultra-cyclists, with exceptions according to each athlete's individual nutrition habits. The ultra-MTBers who drank more, had a higher frequency of food intake. In all participants, increased levels of food, fluid and supplement ingestion tracked across all segments of the race and vice versa. Future studies need to use employ a more accurate method of measuring and recording dietary intake in order to estimate macronutrient, micronutrient, mineral and supplement intake during ultra-endurance races.

\section{Competing interests}

The authors declare that they have no competing interests.

\begin{abstract}
Authors' contributions
All authors have been involved in writing, drafting and revising the manuscript. DCH and BK developed the objectives of the study and intervention. DCH conceived, designed, coordinated the study, collected all data and drafted the manuscript. IT helped with statistical analysis. BK, TR, MS and VCH participated in the design of the study and revised the manuscript. MS helped with translation and the extensive correction of the whole text. All authors read and approved the final manuscript.
\end{abstract}

\section{Acknowledgements}

We thank the organizers of the 'Czech championship 24-hour MTB 2012' in Jihlava 2012 and the 24-hour race 'Bike Race Marathon MTB Rohozec' in Liberec 2012 for their generous support. A special thank goes to all racers participating in our research.

\section{Author details}

${ }^{1}$ Centre of Sports Activities, Brno University of Technology, Brno, Czech Republic. ${ }^{2}$ Institute of Primary Care, University of Zurich, Zurich, Switzerland. ${ }^{3}$ Gesundheitszentrum St. Gallen, St. Gallen, Switzerland. ${ }^{4}$ Faculty of Forestry and Wood Sciences, Czech University of Life Sciences, Prague, Czech Republic. ${ }^{5}$ Department of Preventive Medicine, Faculty of Medicine, Masaryk University, Brno, Czech Republic. 'Institute of Technology Tallaght, Dublin, Ireland. 'Facharzt FMH für Allgemeinmedizin, Gesundheitszentrum St. Gallen, Vadianstrasse 26, 9001 St. Gallen, Switzerland.
Received: 21 March 2014 Accepted: 21 November 2014

Published: 9 December 2014

\section{References}

Applegate EA (1991) Nutritional considerations for ultraendurance performance. Int J Sport Nutr 1:118-126

Astorino TA, Cottrell T, Talhami Lozano A, Aburto-Pratt K, Duhon J (2012) Effect of caffeine on RPE and perceptions of pain, arousal, and pleasure/displeasure during a cycling time trial in endurance trained and active men. Physiol Behav 106:211-217

Berardi JM, Price TB, Noreen EE, Lemon PW (2006) Postexercise muscle glycogen recovery enhanced with a carbohydrate-protein supplement. Med Sci Sports Exerc 38:1106-1113

Berardi JM, Noreen EE, Lemon PW (2008) Recovery from a cycling time trial is enhanced with carbohydrate-protein supplementation vs. isoenergetic carbohydrate supplementation. J Int Soc Sports Nutr 5:1-11

Bescós R, Dodríguez FA, Iglesias X, Knechtle B, Benítez A, Marina M, Padullés JM, Torrado P, Vazquez J, Rosemann T (2012a) Nutritional behavior of cyclists during a 24-hour team relay race: a field study report. J Int Soc Sports Nutr 9:3

Bescós R, Dodríguez FA, Iglesias X, Benítez A, Marina M, Padullés JM, Torrado P, Vázquez J, Knechtle B (2012b) High energy deficit in an ultraendurance athlete in a 24-hour ultracycling race. BUMC Proceedings 25:124-128

Bircher S, Enggist A, Jehle T, Knechtle B (2006) Effects of an extreme endurance race on energy balance and body composition - a case study. J Sports Med 5:154-162

Breen L, Tipton KD, Jeukendrup AE (2010) No effect of carbohydrate-protein on cycling performance and indices of recovery. Med Sci Sports Exerc 42:1140-1148

Burke LM (2002) Feeding ultra-endurance athletes: An interview with Dr. Helen O'Connor and Gregory Cox. Int J Sport Nutr Exerc Metab 12:490-494

Burke LM (2008) Caffeine and sports performance. J Appl Physiol Nutr Metab 33:1319-1334

Burke LM, Cox GR, Cummings NK, Desbrow B (2001) Guidelines for daily carbohydrate intake. Do athletes achieve them? Sports Med 31:267-299

Burke LM, Hawley JA, Wong SH, Jeukendrup AE (2011) Carbohydrates for training and competition. J Sports Sci 29:S17-S27

Cano NJ, Fouque D, Neverve XM (2006) Application of branched-chain amino acids in human pathological states: renal failure. J Nutr 136:S299-S307

Cermak NM, van Loon LJ (2013) The use of carbohydrates during exercise as an ergogenic aid. Sports Med 43:1139-1155

Clark N, Tobin J, Ellis C (1992) Feeding the ultraendurance athlete:practical tips and a case study. J Am Diet Assoc 92:1258-1262

Conway KJ, Orr R, Stannard SR (2003) Effect of a divided caffeine dose on endurance cycling performance, postexercise urinary caffeine concentration, and plasma paraxanthine. J Appl Physiol 94:1557-1562

Cox GR, Desbrow B, Montgomery PG, Anderson ME, Bruce CR, Macrides TA, Martin DT, Moquin A, Roberts A, Hawley JA, Burke LM (2002) Effect of different protocols of caffeine intake on metabolism and endurance performance. J Appl Physiol 93:990-999

Desbrow B, Biddulph C, Devlin B, Grant GD, Anoopkumar-Dukie S, Leveritt MD (2012) The effects of different doses of caffeine on endurance cycling time trial performance. J Sports Sci 30:115-120

Eberle S (2007) Endurance Sports Nutrition. IL. Human Kinetics, Champaign

Enqvist JK, Mattsson CM, Johansson PH, Brink-Elfegoun T, Bakkman L, Ekblom BT (2010) Energy turnover during 24 hours and 6 days of adventure racing. J Sports Sci 28:947-955

Fernstrom JD (2005) Branched-chain amino acids and brain function. J Nutr 135:1539S-1546S

Francescato MP, Di Prampero PE (2002) Energy expenditure during an ultra-endurance cycling race. J Sports Med Phys Fit 42:1-7

Frohnauer A, Schwanke M, Kulow W, Kohler G, Knechtle B (2008) No effects of pre race supplementation with vitamins and minerals on performance in an ultra-endurance race. Pakistan J Nutr 7:283-286

Ganio MS, Klau JF, Casa DJ, Armstrong LE, Maresh CM (2009) Effects of caffeine on sport-specific endurance performance: A systematic review. J Strength Cond Res 23:315-324

Goldstein ER, Ziegenfuss T, Kalman D, Kreider R, Campbell B, Wilborn C, Taylor L, Willoughby D, Stout J, Graves BS, Wildman R, Ivy JL, Spano M, Smith AE, Antonio J (2010) International society of sports nutrition position stand: Caffeine and performance. J Int Soc Sports Nutr 7:5 
Gomez-Cabrera MC, Domenech E, Romagnoli M, Arduini A, Borras C, Pallardo FV, Sastre J, Viña (2008) Oral administration of vitamin C decreases muscle mitochondrial biogenesis and hampers training-induced adaptations in endurance performance. Am J Clin Nutr 87:142-149

Hargreaves M, Hawley JA, Jeukendrup A (2004) Pre-exercise carbohydrate and fat ingestion: effects on metabolism and performance. J Sports Sci 22:31-38

Havemann L, Goedecke JH (2008) Nutritional practices of male cyclists before and during an ultraendurance event. Int J Sport Nutr Exerc Metab 18:551-566

Hawley JA, Hopkins WG (1995) Aerobic glycolytic and aerobic lipolytic power systems. Sports Med 19:240-250

Hew-Butler T, Verbalis JG, Noakes TD (2006) Updated fluid recommendation: position statement from the International marathon medical directors association (IMMDA). Clin J Sport Med 16:283-292

Jeukendrup AE (2004) Carbohydrate intake during exercise and performance. Nutrients 20:669-677

Jeukendrup AE (2010) Carbohydrate and exercise performance: The role of multiple transportable carbohydrates. Curr Opin Clin Nutr Metab Care 13:452-457

Jeukendrup AE (2011) Nutrition for endurance sports: Marathon, triathlon, and road cycling. J Sports Sci 29:91-99

Jeukendrup AE (2014) A step towards personalized sports nutrition: carbohydrate intake during exercise. Sports Med 44:S25-S33

Kimber NE, Ross JJ, Mason SL, Speedy DB (2002) Energy balance during an ironman triathlon in male and female triathletes. Int J Sport Nutr Exerc Met 12:47-62

Knechtle B (2013) Nutrition in ultra-endurance rating - aspects of energy balance, fluid balance and exercise-associated hyponatremia. Med Sport 17:200-210

Knechtle B, Knechtle P, Müller G, Zwyssig D (2003) Energy turnover during a 24 hour cycling race: changes in body mass and adipose subcutaneous tissue. Österreichisches Journal für Sportmedizin 33:11-18

Knechtle B, Enggist A, Jehle T (2005) Energy turnover at the Race across America (RAAM) - A case report. Int J Sports Med 26:499-503

Knechtle B, Pitre J, Chandler C (2007) Food habits and use of supplements in ultra-endurance cyclists - the Race Across America (RAAM) 2006. Schweiz Z Sportmed 55:102-106

Knechtle B, Knechtle P, Schulze I, Kohler G (2008) Vitamins, minerals and race performance in ultra-endurance runners - Deutschlandlauf 2006. Asia Pac $J$ Clin Nutr 17:194-198

Knechtle B, Mrazek C, Wirth A, Knechtle P, Rüst CA, Senn O, Rosemann T, Imoberdorf R, Ballmer P (2012) Branched-chain amino acid supplementation during a 100-km ultra-marathon - A randomized controlled trial. J Nutr Sci Vitaminol 58:36-44

Kreider RB (1991) Physiological considerations of ultraendurance performance. Int J Sport Nutr 1:3-27

Lambert EV, Goedecke JH (2003) The role of dietary macronutrients in optimizing endurance performance. Curr Sports Med Rep 4:194-201

Lambert EV, Speechly DP, Dennis SC, Noakes TD (1994) Enhanced endurance in trained cyclists during moderate intensity exercise following 2 weeks adaptation to a high fat diet. Eur J Appl Physiol Occup Physiol 69:287-293

Laursen PB, Rhodes EC (2001) Factors affecting performance in an ultraendurance triathlon. Sports Med 31:195-209

Linderman JK, Laubach LL (2004) Energy balance during 24 hours of treadmill running. J Exerc Physiol 7:37-44

Matsumoto K, Koba T, Hamada K, Tsujimoto H, Mitsuzono R (2009) Branchedchain amino acid supplementation increases the lactate threshold during an incremental exercise test in trained individuals. J Nutr Sci Vitaminol 55:52-58

Maughan RJ, Greenhaff PL, Hespel P (2011) Dietary supplements for athletes: Emerging trends and recurring themes. J Sports Sci 29:57-66

Meesen R, Roelands B, Spriet LL (2013) Caffeine, exercise and the brain. Nestle Nutr Inst Workshop Ser 76:1-12

Millard-Stafford M, Childers WL, Conger SA, Kampfer AJ, Rahnert JA (2008) Recovery nutrition: timing and composition after endurance exercise. Curr Sports Med Rep 7:193-201

Nielsen FH, Lukaski HC (2006) Update on the relatioship between magnesium and exercise. Magnesium Res 19:180-189

Ormsbee MJ, Bach CW, Baur DA (2014) Pre-exercise nutrition: the role of macronutrients, modified starches and supplements on metabolism and endurance performance. Nutrients 6:1782-1808

Paulsen G, Cumming KT, Holden G, Hallén J, Rønnestad BR, Sveen O, Skaug A, Paur I, Bastani NE, Østgaard HN, Buer C, Midttun M, Freuchen F, Wiig H,
Ulseth ET, Garthe I, Blomhoff R, Benestad HB, Raastad T (2014) Vitamin C and $\mathrm{E}$ supplementation hampers cellular adaptation to endurance training in humans: a double-blind randomized controlled trial. J Physiol 592(Pt 8):1887-1901

Peters EM (2003) Nutritional aspects in ultra-endurance exercise. Curr Opin Clin Nutr Metab Care 6:427-434

Pfeiffer B, Stellingwerff T, Hodgson AB, Randell R, Pöttgen K, Res P, Jeukendrup AE (2012) Nutritional intake and gastrointestinal problems during competitive endurance events. Med Sci Sports Exerc 44:344-351

Phinney SD, Bistrian BR, Evans WJ, Gervino E, Blackburn GL (1983) The human metabolic response to chronic ketosis without caloric restriction: preservation of submaximal exercise capability with reduced carbohydrate oxidation. Metabolism 32:769-776

Pink WC, Keong CC, Bandyopadhyay A (2010) Effects of acute supplementation of caffeine on cardiorespiratory responses during endurance running in a hot \& humid climate. Indian J Med Res 132:36-41

Ranchordas MK (2012) Nutrition for adventure racing. Sports Med 42:915-927

Rehrer NJ (2001) Fluid and electrolyte balance in ultra-endurance sport. Sports Med 31:701-715

Rowland DS, Hopkins WG (2002) Effects of high-fat, high-carbohydrate, and high-protein meals on metabolism and performance during endurance cycling. Int J Sport Nutr Exerc 12:318-335

Saunders MJ, Kane MD, Todd MK (2004) Effects of a carbohydrate-protein beverage on cycling endurance and muscle damage. Med Sci Sports Exerc 36:1233-1238

Saunders MJ, Luden ND, Herrick JE (2007) Consumption of an oral carbohydrate-protein gel improves cycling endurance and prevents postexercise muscle damage. J Strength Cond Res 21:678-684

Sharp CP, Pearson DR (2010) Amino acid supplements and recovery from high-intensity resistence training. J Strength Cond Res 24:1125-1130

Shimomura Y, Murakami T, Nakai N, Nagasaki M, Harris RA (2004) Exercise promotes BCAA catabolism: effects of BCAA supplementation on skeletal muscle during exercise. J Nutr 134:S1583-S1587

Singh A, Evans P, Gallagher KL, Deuster PA (1993) Dietary interest and biochemical profiles of nutritional status of ultramarathoners. Med Sci Sports Exerc 25:328-334

Stadheim HK, Kvamme B, Olsen R, Drevon CA, Ivy JL, Jensen J (2013) Caffeine increases performance in cross-country double-poling time trial exercise. Med Sci Sports Exerc 45:2175-2183

Starling RD, Trappe TA, Parcell AC, Kerr CG, Fink WJ, Costill DL (1997) Effects of diet on muscle triglyceride and endurance performance. J Appl Physiol 82:1185-1189

Stellingwerff T, Cox GR (2014) Systematic review: Carbohydrate supplementation on exercise performance or capacity of varying durations. Appl Physiol Nutr Metab 39:998-1011

Stuempfle KJ, Hoffman MD, Weschler LB, Rogers IR, Hew-Butler T (2011) Race diet of finishers and non-finishers in a 100 mile $(161 \mathrm{~km})$ mountain footrace. J Am Coll Nutr 30:529-535

Tarnopolsky M (2004) Protein requirements for endurance athletes. Nutrition 20:662-668

Valentine RJ, Saunders MJ, Todd MK, St Laurent TG (2008) Influence of carbohydrate-protein beverage on cycling endurance and indices of muscle disruption. Int J Sport Nutr Exerc Metab 18:363-378

White JA, Ward C, Nelson H (1984) Ergogenic demands of a 24 hour cycling event. Br J Sport Med 18:165-171

Williams MH (2005) Dietary supplements and sports performance minerals. J Int Soc Sports Nutr 2:43-49

\section{doi:10.1186/2193-1801-3-715}

Cite this article as: Chlíbková et al:: Nutrition habits in 24-hour mountain bike racers. SpringerPlus 2014 3:715. 